\title{
Consensus of double integrator multi-agents under communication delay
}

\author{
Alexandre Seuret* Dimos V. Dimarogonas ${ }^{* *}$ Karl H. Johansson *** \\ * NeCS Team, Automatic Control Department of Grenoble GIPSA-Lab, UMR \\ CNRS 5216. E-mail: alexandre.seuretegipsa-lab.inpg.fr \\ ** Laboratory for Information and Decision Systems, Massachusetts Institute \\ of Technology, Cambridge, MA, U.S.A. \{ddimar@mit.edu\} \\ *** ACCESS Linnaeus Center, Royal Institute of Technology, Stockholm, \\ Sweden. kallej@kth.se
}

\begin{abstract}
This paper deals with the consensus problem under network induced communication delays. It is well-known that introducing a delay generally leads to a reduce of the performance or to instability. Thus, investigating the impact of time-delays in the consensus problem is an important issue. Another important issue is to obtain an estimate of the convergence rate, which is not straightforward when delays appear in the network. In this paper, the agents are modelled as double integrator systems. It is assumed that each agent receives instantaneously its own output information but receives the information from its neighbors after a constant delay. A stability criterion is provided based on Lyapunov-Krasovskii techniques and is expressed in terms of LMI. An expression of the consensus equilibrium which depends on the delay and on the initial conditions taken in an interval is derived. The results are supported through several simulations for different network symmetric communication schemes.
\end{abstract}

\section{INTRODUCTION}

Distributed algorithms for cooperative control of multi-agent systems is a field that has gained increasing attention in the last few years, due to its applications in multi-robot systems OlfatiSaber and Murray [2004], averaging in communication networks Xiao and Boyd [2003] and formation control Dimarogonas and Kyriakopoulos [2008]. Several results have appeared in recent literature that consider systems with different motion models, symmetry of communication and network interactions. A recent review of the vast literature in the field can be found in Olfati-Saber and Murray [2004].

In this paper, a particular case of the consensus problem is treated, in which the information exchange between the communicating agents has inherit time-delays. In particular, each agent is assumed to have access to the information of its own state with no delays, but can only consider delayed information of the states of its neighbors. The delays of the proposed controller model various phenomena of networked systems such as transmission delays on the transfer of data between each agent and its neighbors, packet losses in wireless communication networks and inaccurate sensor measurements. Moreover, delays can result from sampling. As shown in Fridman et al. [2004], a sampled signal can be seen as a delayed signal with a particular delay $\tau(t)=t-t_{k}$, which is discontinuous and whose derivative is equal to 1 almost every time. As it is not clear that all the agents have synchronized clocks, the assumption that these sampling delays are known is not satisfied in a general case.

In contrast to our previous work Seuret et al. [2008], where we considered the simple case of single integrator agents, the agents are assumed to obey a double integrator model. This

\footnotetext{
* This work was supported by the European Project FeedNetBack : http://www.feednetback.eu/.
}

model fits the behavior of real robotic agents more naturally, since such mechanical systems are controlled in most cases through their acceleration and not their velocity. Moreover, several robotic systems can be reduced to a double integrator via a transformation in their control law. In this paper, we provide stability conditions for consensus of multiple double integrator agents using Lyapunov-Krasovskii techniques which are given in terms of LMI. The communication topology is asymmetric and the symmetric case is treated as a special case of the main theory. Note that the case of single integrator agents with time delays was also treated in Moreau [2004], Moreau [2005],Olfati-Saber et al. [2007].

The rest of the paper is organized as follows: Section II includes the necessary background on consensus and time-delay systems and presents the problem treated in this paper. The stability analysis of the closed-loop system is given in Section III which includes both the cases of asymmetric and symmetric communication topologies. Section IV includes illustrating simulation examples while Section V summarizes the results of the paper and indicates current research efforts.

\section{PROBLEM STATEMENT}

We first review the original non delayed consensus problem for $N \in \mathbb{N}$ agents with fixed but non necessarily symmetric communication links. The open-loop dynamics are given by:

$$
\ddot{x}_{i}(t)=u_{i}(t), \quad i \in\{1, \ldots, N\} .
$$

The consensus control law with no time delays is given by

$$
u_{i}(t)=-\dot{x}_{i}(t)+\sum_{j \in \mathscr{N}_{i}} a_{i j}\left(x_{j}(t)-x_{i}(t)\right), \quad i \in\{1, \ldots, N\} .
$$

where $\mathscr{N}_{i}$ represents the set of agents which are connected to agent $i$ and is called agent $i$ 's communication set. The gains $a_{i j}$ are positive scalar. Note that the communication is not necessarily symmetric, which means $a_{i j} \neq a_{j i}$. 
The closed-loop system is written in stack vector form as

$$
\ddot{x}=-\dot{x}-L x
$$

where $x=\left[x_{1}, \ldots, x_{N}\right]^{T}$ is the stack vector of all agents' states and $L$ is the Laplacian matrix Godsil and Royle [2001] of the communication graph $G$ of the network, which is defined based on the communication sets $\mathscr{N}_{i}$.

A brief background on the construction of the Laplacian matrix is given in the sequel. For the graph $G$ with $N$ vertices and edge set given by $E=\left\{(i, j): j \in \mathscr{N}_{i}\right\}$ the adjacency matrix $A=A(G)=\left(a_{i j}\right)$ is the $N \times N$ matrix given by $a_{i j}=1$, if $(i, j) \in E$ and $a_{i j}=0$, otherwise. If there is an edge connecting two vertices $i, j$, i.e. $(i, j) \in E$, then $i, j$ are called adjacent. If there is a path between any two vertices of the graph $G$, then $G$ is called strongly connected in the case of directed, and simply connected in the case of undirected graphs. The degree $d_{i}$ of vertex $i$ is defined as the number of its neighboring vertices, i.e. $d_{i}=\# j:(i, j) \in E$. Let $\Delta$ be the $N \times N$ diagonal matrix of $d_{i}$ 's. The Laplacian of $G$ is the matrix $L=\Delta-A$. For an undirected graph the Laplacian matrix is symmetric positive semidefinite. When the directed graph is strongly connected, the Laplacian has a single zero eigenvalue and the corresponding eigenvector is the vector of ones, $\overrightarrow{\mathbf{1}}$. This result was established in Olfati-Saber et al. [2007]. For the case of undirected graphs, a necessary and sufficient condition for zero to be a simple eigenvalue of the Laplacian matrix, is that the undirected graph is connected.

In this paper, the following problem in considered. Each agent $i$ has access to the value of $x_{i}$ through embedded sensors. Thus the state $x_{i}$ is available to agent $i$ at every time $t$ without any delay. However, the data coming from the other agents $j \in \mathscr{N}_{i}$ are received by agent $i$ after a time-delay caused by the various reasons given in the introduction. Consider further as an approximation that all the communication delays are constant and equal to $\tau$ which can be perceived as an average delay. The control law for agent $i$ is then given by:

$$
\ddot{x}_{i}(t)=-\dot{x}_{i}(t)+\sum_{j \in \mathscr{N}_{i}} a_{i j}\left(x_{j}(t-\tau)-x_{i}(t)\right) \quad i \in\{1, \ldots, N\},
$$

Remark 1. The convergence of the rendezvous algorithm with 'heterogenous' delays (i.e. in (4) the delay is $\tau_{i j}$ instead of $\tau$ ) has already been studied in Munz et al. [2008]. However the analysis of the agreement point has not been characterized.

Considering $x(t)=\left[x_{1}(t), . ., x_{N}(t)\right]^{T}$, the closed-loop system is given by:

$$
\ddot{x}(t)=-\dot{x}(t)-\Delta x(t)+A x(t-\tau),
$$

where $A$ is the adjacency matrix of the communication graph and $\Delta$ is the diagonal matrix of the $d_{i}$ 's.

\section{STABILITY OF CONSENSUS UNDER COMMUNICATION DELAY USING LKF'S}

This section focuses on the convergence of the consensus for problem (5). A new approach to the stability of the consensus under communication delay is provided in this section. An expression of the consensus equilibrium is given. More especially, this expression shows the influence of the initial conditions and the delay value on the consensus set.

\subsection{Definition of an appropriate model}

Knowing that the vector $\overrightarrow{\mathbf{1}}$ is an eigenvector associated to the eigenvalue 0 of the Laplacian matrix, it is possible to find a change of coordinates $x=W z$ such that

$$
U(-\Delta+A) W=\left[\begin{array}{cc}
B & 0 \\
0^{T} & 0
\end{array}\right],
$$

where, in the case of strongly connected graphs, $U=\left[\begin{array}{cc}U_{1}^{T} & U_{2}^{T}\end{array}\right]^{T}$ $=W^{-1}$ and $U_{2}=(U)_{N}$. In the case of a symmetric matrix $A$, $B \in \mathbb{R}^{(N-1) \times(N-1)}$ can be the diagonal matrix of the eigenvalues $\lambda_{2}, \ldots, \lambda_{N}$ of the Laplacian. If $L$ represents a disconnected graph, the dimension of the vectors $z_{1}$ and $z_{2}$ changes. In the sequel, the communication graph is assumed to be strongly connected. The next lemma shows an appropriate way to rewrite (5) based on the properties of $L$.

Lemma 1. Assume that there exists $\mu>0$ such that $\Delta=\mu I$. The consensus problem (5) can be rewritten using $z_{1} \in R^{N-1}$, $z_{2} \in R$ and the matrix $B$ is given in (6):

$$
\begin{array}{r}
\ddot{z}_{1}(t)=-\dot{z}_{1}(t)-\mu z_{1}(t)+(B+\mu I) z_{1}(t-\tau), \\
\ddot{z}_{2}(t)=-\dot{z}_{2}(t)-\mu z_{2}(t)+\mu z_{2}(t-\tau),
\end{array}
$$

Proof. ¿From equation (6), equation (5) can be rewritten using the Leibnitz formula as

$$
\begin{aligned}
& \ddot{z}_{1}(t)=-\dot{z}_{1}(t)+B z_{1}(t)-A_{1}^{\prime} \int_{t-\tau}^{t} \dot{z}(s) d s, \\
& \ddot{z}_{2}(t)=-\dot{z}_{2}(t)+A_{2}^{\prime} \int_{t-\tau}^{t} \dot{z}(s) d s .
\end{aligned}
$$

where $\left[A_{1}^{\prime T} A_{2}^{\prime T}\right]^{T}=U A W$ and $A_{2}^{\prime}=(U A W)_{N}$. The system is split into two equations where $z_{1}$ contains the $N-1$ first components of $z$ and $z_{2}$ is equal to the last component of $z$. Noting that $U A W=U(-\Delta+A) W+U \Delta W$ and using the assumption on $\Delta=\mu I$, (6) leads to

$$
\left[\begin{array}{l}
A_{1}^{\prime} \\
A_{2}^{\prime}
\end{array}\right]=U(-\mu I+A) W+\mu I=\left[\begin{array}{cc}
B+\mu I & 0 \\
0^{T} & \mu
\end{array}\right] .
$$

Noting that $\int_{t-\tau}^{t} \dot{z}_{i}(s) d s=z_{i}(t)-z_{i}(t-\tau)$, system (8) can be rewritten as (7).

The consensus problem is now expressed in an appropriate form to perform stability criteria. Note that the variable $z_{2}$ is defined by $z_{2}(t)=U_{2} x(t)$. In the case of a symmetric network, the matrix $W$ is an orthogonal matrix which means $U=W^{T}$. Then if the last column of $W$ is $\alpha \overrightarrow{\mathbf{1}}$, then $U_{2}=1 /(\alpha N) \overrightarrow{\mathbf{1}}$, which means that $z_{2}$ corresponds to the average of the position of all agents. This does not hold for asymmetric communication network. In the case of disconnected graph, the same lemma holds but the dimension of $z_{2}$ increases. In the sequel, the communication graph is assumed strongly connected.

Lemma 2. Assume that there exists $\mu>0$ such that $\Delta=\mu I$. The system defined in (7b) is stable for any delay $\tau$ and converges to

$$
z_{2 e q}=\lim _{s \rightarrow 0} s \frac{(s+1) z_{2}(0)+\dot{z}_{2}(0)+\mu \int_{-\tau}^{0} z_{2}(u) e^{-(u+\tau) s} d s}{s^{2}+s+\mu\left(1-e^{-\tau s}\right)}
$$

Proof. The analysis is based on a frequency approach. The Laplace transform of system $(7 b)$ is

$$
\begin{aligned}
s^{2} Z_{2}(s) & =s z_{2}(0)+\dot{z}_{2}(0)-s Z_{2}(s)+z_{2}(0)-\mu Z_{2}(s) \\
& +\mu e^{-\tau s} Z_{2}(s)+\mu \int_{-\tau}^{0} z_{2}(u) e^{-(u+\tau) s} d u .
\end{aligned}
$$


Thus we have

$$
Z_{2}(s)=\frac{(s+1) z_{2}(0)+\dot{z}_{2}(0)+\mu \int_{-\tau}^{0} z_{2}(u) e^{-(u+\tau) s} d s}{s^{2}+s+\mu\left(1-e^{-\tau s}\right)}
$$

The stability of the equation in $z_{2}$ is analyzed with a pseudodirect method to time delay systems (see paragraph 2.2.2 in $\mathrm{Gu}$ et al. [2003]. Consider the characteristic quasi polynomial $a\left(s, e^{-\tau s}\right)=s^{2}+s+\mu\left(1-e^{-\tau s}\right)$, which allows analyzing the stability of the system in $z_{2}$. We first notice that the system is stable at $\tau=0$. Consider now the bilinear transformation:

$$
z=\frac{1-T s}{1+T s}, \quad T>0
$$

Consider the polynomial:

$$
C(T, s)=(1+T s)\left(s^{2}+s+\mu(1-(1-T s) /(1+T s))\right.
$$

¿From Gu et al. [2003], if the roots of the polynomial $C(T, s)$ for all $T>0$ and those of $a(s,-1)$ are on the left-hand side of the complex plane, then the original time-delay system is stable. Note that

$$
C(T, s)=s\left(T s^{2}+(1+T) s+1+2 \mu T\right)
$$

has one zero pole and the others lie in the left-hand side of the complex plane (since all the terms are positive). This means that the roots of $C$ are stable. Noting that the roots of $a(s,-1)=s^{2}+$ $s+2 \mu$ are also stable, the system (7b) is stable and the final equilibrium of $z_{2}$ is given by the theorem of the final value which leads to (9).

\subsection{Stability of the consensus problem with communication delay}

Consider the consensus problem with communication delay (5) rewritten in the form of (7). Provided that the graph is strongly connected, the consensus problem (5) can be rewritten as (7). Regarding the stability of $z_{1}$, consider the state-space representation:

$$
\dot{\bar{z}}_{1}(t)=C_{0} \bar{z}_{1}(t)+C_{1} \bar{z}_{1}(t-\tau)
$$

where $\bar{z}_{1}(t)=\left[\begin{array}{c}z_{1}^{T}(t) \\ \dot{z}_{1}^{T}(t)\end{array}\right]$ and $C_{0}=\left[\begin{array}{cc}0 & I \\ -\mu I & -I\end{array}\right]$ and $C_{1}=\left[\begin{array}{cc}0 & 0 \\ B+\mu I & 0\end{array}\right]$. The following theorem ensures the stability of such a system:

Theorem 1. Consider any Laplacian matrix $L=\mu I-A$ which represents a strongly connected graph. Consider the associated consensus problem (5) with a constant delay $\tau$. If there exist positive definite matrices $P, S$ and $R$ in $R^{n \times n}$, and a matrix $Y \in R^{n \times n}$ such that the following LMIs are satisfied:

$$
\begin{gathered}
\Psi(\mu, B)=M_{2}^{T} P M_{1}+M_{1}^{T} P M_{2}+M_{2}^{T} S M_{2}-M_{3}^{T} S M_{3} \\
+\tau M_{1}^{T} R M_{1}-M_{4}^{T} R M_{4} / \tau<0,
\end{gathered}
$$

where

$$
M_{1}=\left[\begin{array}{ll}
C_{0} & C_{1}
\end{array}\right], M_{2}=\left[\begin{array}{ll}
I & 0
\end{array}\right], M_{3}=\left[\begin{array}{ll}
0 & I
\end{array}\right], M_{4}=\left[\begin{array}{ll}
I & -I
\end{array}\right]
$$

Then (5) converges asymptotically to the equilibrium given by $x_{e q}=U_{2} x(0) \overrightarrow{\mathbf{1}}$.

Proof. Consider the initial consensus problem (5) under a symmetric or non-symmetric communication network inducing a constant delay $\tau$. There exists a change of coordinates $z=$ $W x$ ( $W$ is an orthogonal matrix in the symmetric case or a non singular matrix in the non symmetric case) such that the problem can be rewritten as (7). The first part of the proof consists in ensuring the stability of the reduced-order variable
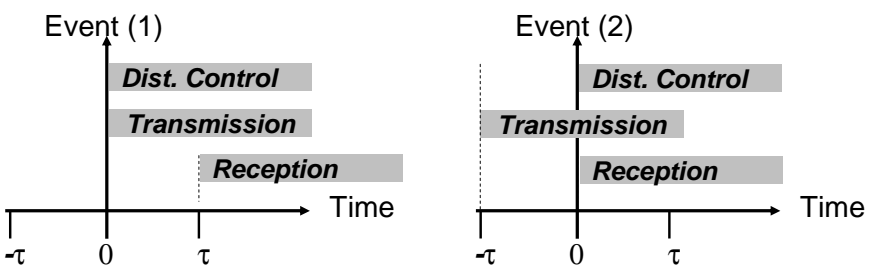

Fig. 1. Protocole to ensure that the agents reach the average of the initial position

$z_{1}$. To do so, consider the stability analysis of system (7a) with the following LKF

$$
\begin{aligned}
V\left(z_{1}\right)= & \bar{z}_{1}^{T}(t) P \bar{z}_{1}(t)+\int_{t-\tau}^{t} \bar{z}_{1}^{T}(s) S \bar{z}_{1}(s) d s \\
& +\int_{-\tau}^{0} \int_{t+\theta}^{t} \dot{\bar{z}}_{1}^{T}(s) R \dot{\bar{z}}_{1}(s) d s d \theta,
\end{aligned}
$$

Using the matrices $M_{i}$ with $i=1,2,3$, the time-derivative of the functional along the trajectories of (14) is expressed as follows:

$$
\begin{array}{r}
\dot{V}\left(z_{1}\right)=\xi^{T}(t)\left[2 M_{2}^{T} P M_{1}+M_{2}^{T} S M_{2}-M_{3}^{T} S M_{3}\right. \\
\left.+\tau M_{1}^{T} R M_{1}\right] \xi(t)-\int_{t-\tau}^{t} \dot{\bar{z}}_{1}^{T}(s) R \dot{\bar{z}}_{1}(s) d s
\end{array}
$$

The Jensen inequality gives a suitable bound for the last term of (12):

$$
\begin{aligned}
-\int_{t-\tau}^{t} \dot{\bar{z}}_{1}^{T}(s) R \dot{\bar{z}}_{1}(s) d s & \leq-\int_{t-\tau}^{t} \dot{\bar{z}}_{1}^{T}(s) d s(R / \tau) \int_{t-\tau}^{t} \dot{\bar{z}}_{1}(s) d s \\
& \leq-\xi^{T}(t) M_{4}^{T}(R / \tau) M_{4} \xi(t)
\end{aligned}
$$

$\dot{V}$ can thus be bounded by $\dot{V}\left(z_{1}\right) \leq \xi^{T}(t) \Psi(\mu, B) \xi(t)$. Then if $\Psi$ is a negative definite matrix, the derivative of the LyapunovKrasovskii functional is also negative definite. Then system (7a) is asymptotically stable.

Note that Theorem 1 is delay dependent. This means that the stability conditions depend on the value of the delay. This allows analyzing the stability of systems which are stable for "small" delays but become unstable for larger delays. In timedelay theory, this functional ensures that if the conditions from Theorem 1 hold for a particular $\tau$, then the system is stable for all constant delays which belong to $[0, \tau]$.

\subsection{Discussion on the effect of the initial conditions}

¿From Theorem 1, it can be seen that the initial conditions have a strong effect on the equilibrium state. The following corollaries consider special setups which affect the averaging properties of the consensus:

Corollary 1. Assume that the initial conditions are: $x(\theta)=$ $0, \forall \theta \in\left[-\tau, 0\left[x(\theta)=x_{0}\right.\right.$ at time 0 . The initial condition on the velocity is $\dot{x}(\theta)=\dot{x}_{0}$. Then the equilibrium is $x_{e q}=U_{2}\left(x_{0}+\right.$ $\left.\dot{x}_{0}\right) /(1+\mu \tau) \overrightarrow{\mathbf{1}}$.

Corollary 2. Assume that the initial conditions are: $x(\theta)=$ $x_{0}, \quad \forall \theta \in[-\tau, 0]$ and $\dot{x}(0)=0$. Then the equilibrium is $x_{e q}=$ $U_{2} x_{0} \overrightarrow{\mathbf{1}}$.

Proof. The result is straightforwardly given by the equation given in Theorem 1. In the two corollaries, the integrals are computed as $\int_{-\tau}^{0} x(u) e^{-(u+\tau) s} d s=0$ and $\mu x(0)\left(1-e^{-\tau s}\right) / s$ respectively. The expressions of the equilibria in each corollary are given by the limit when $s$ goes to zero. 
Corollaries 1 and 2 can be interpreted using Figure 1. The difference between the two behaviors can also be seen in the examples' section. These two corollaries allow for understanding the influence of two different specifications of the initial conditions. More specifically, these cases have a practical implication and allow for considering a simple setup to implement the multiagent system to conserve the averaging properties. Corollary 1 deals with the case when the distributed control is computed at time $t=0$. However because of the delay, no information is available to the controller until the time $t=\tau$. This means that between $t=0$ and $t=\tau$ the system is running in open-loop. At the time instant $t=\tau$, all the required information to implement the distributed control is available.

This means that, at time $t=-\tau$, all agents have to wait (i.e. to be still and $\dot{x}(t)=0$ ) until they receive data from their neighbors. At time $t=0$, the control using non zero initial conditions in $[-\tau, 0]$ is implemented. The initial condition did not change the final equilibrium of the consensus problem compared to the non delayed case. It finally appears that the stability of the multi-agent system is not affected by the delay. However this does not mean that the performances are not modified. The next section is dedicated to the evolution of the convergence rate with respect to the delay.

Remark 2. The two cases described above only cope with two simple and understandable cases of initial conditions. Other particular setups for the initial conditions can also be considered.

\section{CONVERGENCE RATE}

This section focuses on the evolution of the convergence rate with respect to the delay using a time-domain approach. The initial conditions are chosen to satisfy Corollary 2 since they preserve the averaging property of the consensus. Moreover this choice of initial conditions does not add additional dynamics since the variable $z_{2}$ is constant during the simulation. This section focuses on the behavior of the complementary variables. More specifically, the objective is to estimate the exponential convergence rate of the agents to reach a consensus. Consider the definition of exponential stability:

Definition 1. Mondié and Kharitonov [2005] System (7a) is exponentially stable with a decay rate $\delta>0$ if there exists a gain $\gamma \geq 1$ such that the solution $z_{1}\left(t, t_{0}, \phi\right)$ of (7a), starting at time $t_{0}$ from any initial condition $\phi \in \mathscr{C}^{1}$, satisfies $\left|z_{1}\left(t, t_{0}, \phi\right)\right|<$ $\gamma|\phi|{ }_{\tau} e^{-\delta\left(t-t_{0}\right)}$.

Few papers examine the convergence rate of such systems Liu [October 2003], Mondié and Kharitonov [2005], Xu et al. [2006]. In this paper, we focus on the tools developed in Seuret et al. [2004], Xu et al. [2006] to introduce exponential stability criteria and in Fridman and Shaked [2002] to consider one of the less conservative lemmas for the stability of time-delay systems. This section introduces an extension of previous works about the convergence rate of a consensus to the case of double integrator agents.

Theorem 2. Consider the consensus problem defined in (5) with a constant delay $\tau$. If for a given positive scalar $\delta>0$, there exist positive definite matrices $P, S$ and $R$ in $R^{n \times n}$, and a matrix $Y \in R^{n \times n}$ such that the following LMIs are satisfied:

$$
\begin{aligned}
\Psi_{\delta}(\mu, B) & =M_{2}^{T} P M_{5}+M_{5}^{T} P M_{2}+M_{2}^{T} S M_{2}-M_{3}^{T} S M_{3} \\
& +\tau M_{5}^{T} R M_{5}-M_{4}^{T} R M_{4} / \tau<0,
\end{aligned}
$$

where $M_{5}=\left[\begin{array}{ll}C_{0}+\delta I & e^{\delta \tau} C_{1}\end{array}\right]$. Then (5) converges exponentially with decay rate $\delta$ to the equilibrium given by $x_{e q}=$ $U_{2} x(0) \overrightarrow{\mathbf{1}}$.

Proof. Consider the initial consensus problem (5) under a symmetric or non-symmetric communication network inducing a constant delay $\tau$. There exists a change of coordinates $z=W x$ such that the problem can be rewritten as (7). The proof consists first in ensuring the exponential stability of the reduced-order variable $z_{1}$. Exponential stability is analyzed via the change of variables $\bar{z}_{1 \delta}=e^{\delta t} \bar{z}_{1}(t)$ as in Seuret et al. [2004], Xu et al. [2006]. Then, in the case of constant delay, (7a) becomes:

$$
\begin{aligned}
\dot{\bar{z}}_{1 \delta}(t) & =\left(C_{0}+\delta I\right) \bar{z}_{1 \delta}(t)+e^{\delta \tau}(B+\mu I) \bar{z}_{1 \delta}(t-\tau) \\
& =M_{5} \zeta(t)
\end{aligned}
$$

where $\zeta(t)=\left[\begin{array}{ll}\bar{z}_{1 \delta}^{T}(t) & \bar{z}_{1 \delta}^{T}(t-\tau)\end{array}\right]^{T}$. It follows that if $\bar{z}_{1 \delta}$ converges asymptotically to the solution $z_{1 \delta}=0$, then $z_{1}$ exponentially converges to $z_{1}=0$ with the decay rate $\delta$. Note that system (14) is still a linear system with constant delay but the matrix gains have changed. To analyze the stability of (14), consider the following LKF:

$$
\begin{aligned}
V_{\delta}\left(z_{1 \delta}\right) & =\bar{z}_{1 \delta}^{T}(t) P \bar{z}_{1 \delta}(t)+\int_{t-\tau}^{t} \bar{z}_{1 \delta}^{T}(s) S \bar{z}_{1 \delta}(s) d s \\
& +\int_{-\tau}^{0} \int_{t+\theta}^{t} \dot{\bar{z}}_{1 \delta}^{T}(s) R \dot{\bar{z}}_{1 \delta}(s) d s d \theta
\end{aligned}
$$

The end of the proof is similar to the one of Theorem 1 . $\dot{V}_{\delta}$ can be bounded by $\dot{V}_{\delta}\left(z_{1 \delta}\right) \leq \zeta^{T}(t) \Psi_{\delta}(\mu, B) \zeta(t)$. Then if $\Psi_{\delta}$ is a negative definite matrix, the derivative of the LKF is also definite negative. System (14) is asymptotically stable and thus, (7a) is exponentially stable with the decay rate $\delta$. Finally according to Lemma 2 and the change of coordinates defined by $W$ and $U$, the equilibrium is given by Theorem 2 .

Theorem 2 is also delay-dependent. Based on Theorem 2, it is thus possible to maximize the decay rate $\delta$ with respect to the value of $\tau$. However it does not allow for deriving an analytic expression of the relation between $\tau$ and $\delta$. Note that the stability conditions do not depend on the choice of $W$. It is only required that $B$ belongs to $R^{(N-1) \times(N-1)}$.

Remark 3. Note that assuming $\delta=0$ in Theorem 2 we obtain the same conditions as in Theorem 1. This means that the conditions from Theorem 2 can also be used to ensure the asymptotic stability of the multi-agent system.

Remark 4. In the case of time-varying delays in the communication network, system (14) will be defined with time-varying matrices. To overcome this difficulty, the reader can refer to Seuret et al. [2004].

In Olfati-Saber et al. [2007], it was noted that the consensus problem (4) does not preserve the average consensus. Instead of Moreau [2004] and Moreau [2005], the effect that initial conditions and delays have in the resulting consensus equilibrium is explicitly shown. In this article we extended the result which was provided in Seuret et al. [2008] to the case of double integrator agents. In this case, it is clear that the consensus equilibria will also depend on the initial velocity of the agents.

\section{EXAMPLES}

Consider four agents with the initial positions $x(0)=\left[\begin{array}{llll}0 & 5 & 15 & 20\end{array}\right]^{T}$ and two cases of initial velocities $\dot{x}(0)=\left[\begin{array}{lll}1-15 & -5\end{array}\right]^{T}$ (corresponding to Corollary 1) and $\dot{x}(0)=\left[\begin{array}{llll}0 & 0 & 0 & 0\end{array}\right]^{T}$ (Corollary 


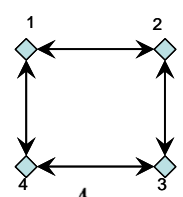

$A_{\mathrm{C}}$

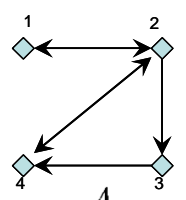

$A_{1}$

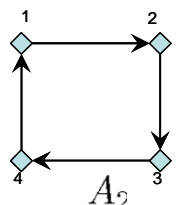

$A_{2}$

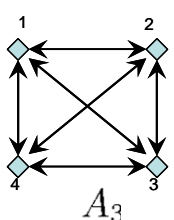

$A_{3}$
Fig. 2. Corresponding graphs of the matrices $A_{i}$ for $i=0, \ldots, 3$.
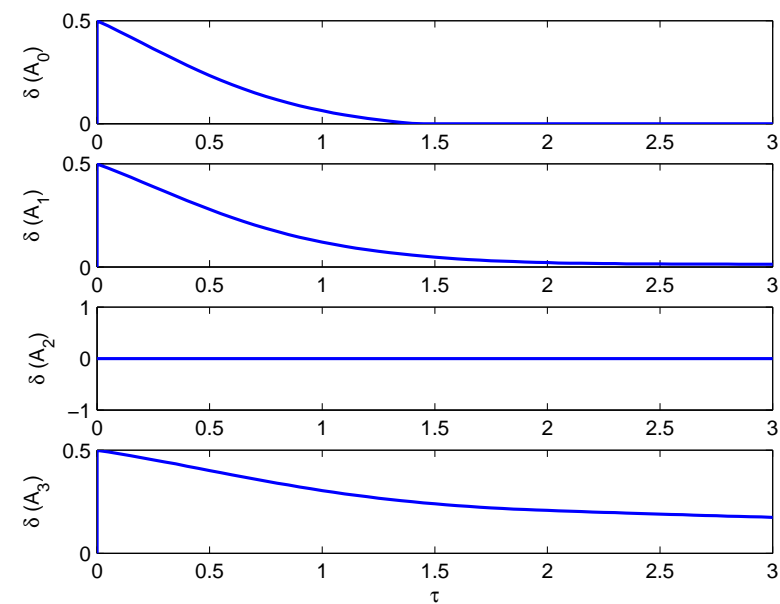

Fig. 3. Convergence rate $\delta$ of the consensus algorithms with $A_{i}$, for $i=0, \ldots, 3$ with respect to the delay $\tau$.

2). Our first objective is to study the convergence rate for four different communication networks and delays. The second objective consists in giving the value of the consensus. The networks are defined with $\mu=1$ and

$$
\begin{gathered}
A_{0}=\left[\begin{array}{cccc}
0 & 0.5 & 0 & 0.5 \\
0.5 & 0 & 0.5 & 0 \\
0 & 0.5 & 0 & 0.5 \\
0.5 & 0 & 0.5 & 0
\end{array}\right], A_{1}=\left[\begin{array}{cccc}
0 & 1 & 0 & 0 \\
0.5 & 0 & 0 & 0.5 \\
0 & 0.5 & 0 & 0.5 \\
0 & 0.5 & 0.5 & 0
\end{array}\right], \\
A_{2}=\left[\begin{array}{llll}
0 & 1 & 0 & 0 \\
0 & 0 & 1 & 0 \\
0 & 0 & 0 & 1 \\
1 & 0 & 0 & 0
\end{array}\right], A_{3}=\left[\begin{array}{cccc}
0 & 1 / 3 & 1 / 3 & 1 / 3 \\
1 / 3 & 0 & 1 / 3 & 1 / 3 \\
1 / 3 & 1 / 3 & 0 & 1 / 3 \\
1 / 3 & 1 / 3 & 1 / 3 & 0
\end{array}\right] .
\end{gathered}
$$

The graphs corresponding to these matrices are given in Figure 2 . Note that $A_{0}$ and $A_{3}$ are symmetric contrary to $A_{1}$ and $A_{2}$. For each graph, the evolution of the convergence rate with respect to the delay is provided in Figure 3.

The performances of the consensus algorithms are very different depending on the communication graph between agents. We would first expect that the performances of the multi-agent systems would be the same for symmetric (and respectively asymmetric) graphs. However the conditions of Theorem 1 for the complete graph $A_{3}$ and the asymmetric graph corresponding to $A_{1}$ can be solved for any delay. Regarding the symmetric graph corresponding to $A_{0}$, the conditions are only satisfied if $\tau \leq 1.41$. Finally, the consensus algorithm with $A_{2}$ is not stable all. This can also be seen by checking the matrix $C_{0}+C_{1}$ which has two eigenvalues on the imaginary axis.

In Seuret et al. [2008], it is shown that a consensus algorithm with single integrator systems, is always stable for any symmetric graph and for any delay. In the case of algorithmes based on
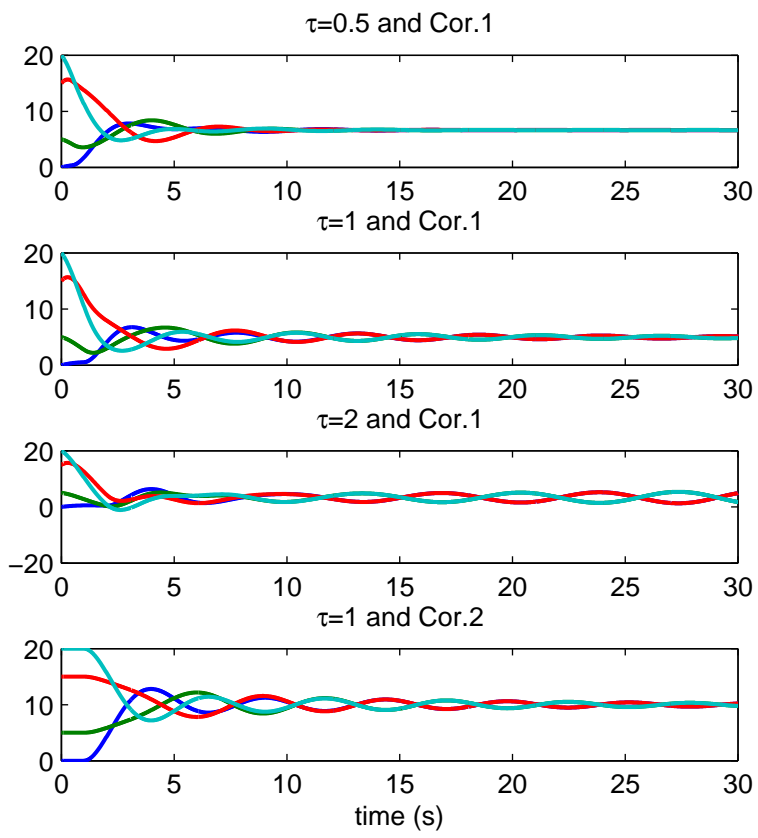

Fig. 4. Simulations of the consensus algorithm with $A_{0}$.
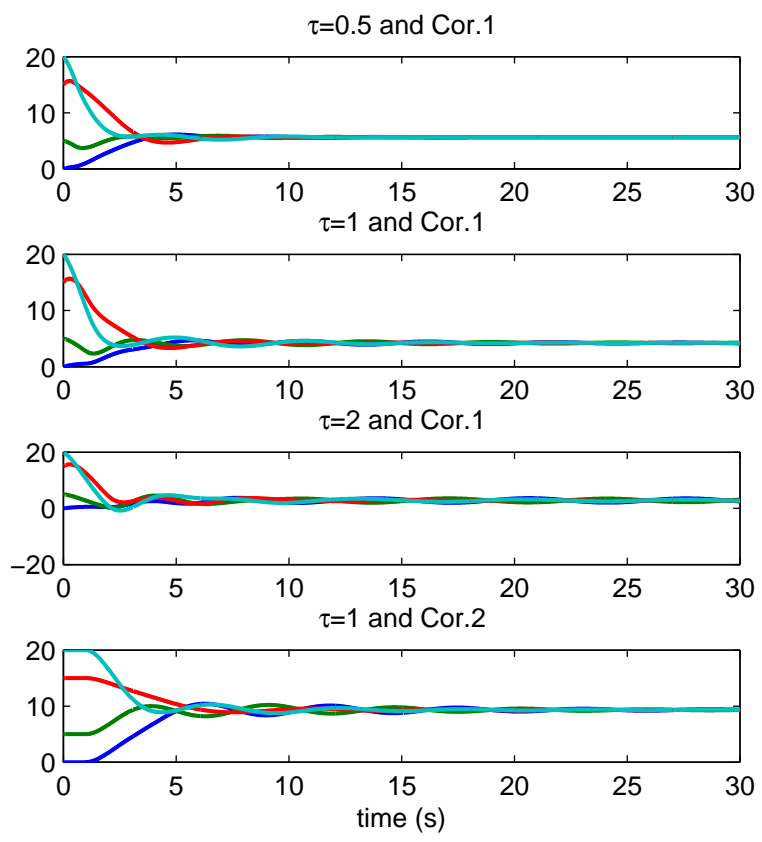

Fig. 5. Simulations of the consensus algorithm with $A_{1}$.

double integrator systems, such general stability conditions can not be provided.

Figures 4, 5 and 6 show the evolution of the four agents for all the networks. The three upper plots correspond to initial conditions following Corollary 1 and with $\tau=0.5,1$ and 2 . The fourth plot shows the multi-agent system state with initial conditions satisfying Corollary 2 and $\tau=1$. In all figures, it can be seen that the usual oscillating behavior of time-delay system appears when the delay is larger. Regarding the case of $A_{1}$, no consensus is achieved for $\tau=2$ and the agents are diverging. 

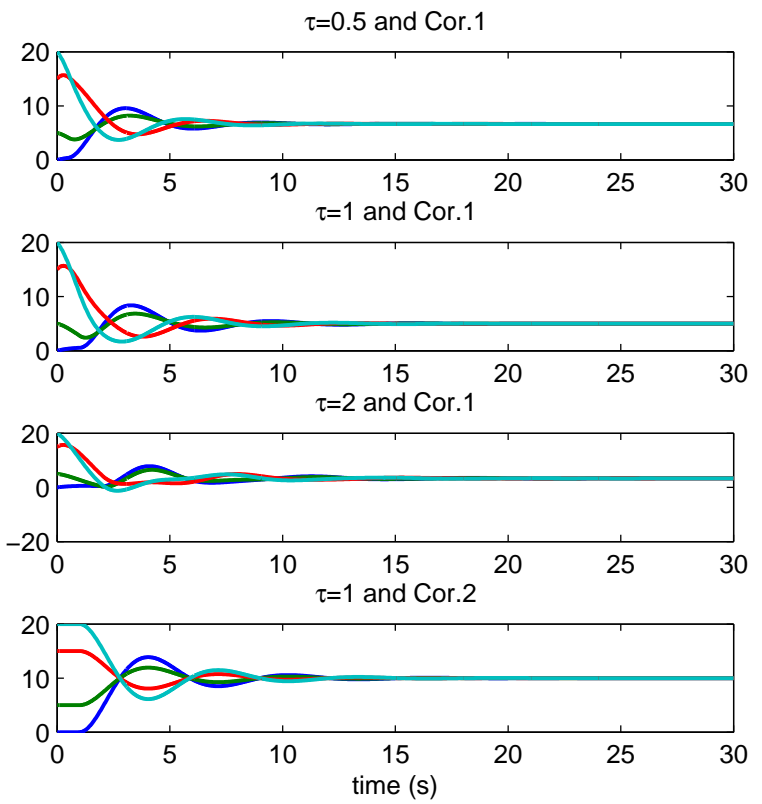

Fig. 6. Simulations of the consensus algorithm with $A_{3}$.

For the three first plots of each figure, the agents are openloop controlled, i.e., they are only driven by the diagonal terms during the interval $[0, \tau]$. As soon as the agents are controlled in closed-loop, i.e., the delayed terms act on the dynamics, the agents can reach a consensus corrupted by the first phase in open-loop. The computation of the consensus equilibrium, using the expression in Theorem 1, for the graphs corresponding to $A_{0}$ and $A_{3}$ leads to $6.66,5,3.33$ and 10 (corresponding respectively to Cor. 1 with $\tau=0.5,1,2$ and Cor. 2 with $\tau=1$ ). Concerning $A_{1}$, the consensus equilibria are $5.64,4.23,2.82$ and 9.33 .

\section{CONCLUSION}

The effect of time-delays in the consensus problem for double integrator agents was studied. The derived result shows that consensus is achieved but its value is strongly dependent both on the value of the delay and on the initial conditions. This time-delay approach allows considering simple symmetric/asymmetric connected/disconnected communication networks. Further research involves consideration of different time-varying delays.

\section{REFERENCES}

D.V. Dimarogonas and K.J. Kyriakopoulos. A connection between formation infeasibility and velocity alignment in kinematic multi-agent systems. Automatica, 44(10):26482654, 2008.

E. Fridman and U. Shaked. An improved stabilization method for linear time-delay systems. IEEE Transactions on Automatic Control, 47(11):1931-1937, 2002.

E. Fridman, A. Seuret, and J.-P. Richard. Robust sampleddata stabilization of linear systems: An input delay approach. Automatica, 40(8):1141-1446, 2004.

C. Godsil and G. Royle. Algebraic Graph Theory. Springer, 2001.

K. Gu, V.-L. Kharitonov, and J. Chen. Stability of time-delay systems. Birkhauser, 2003.
P.-L. Liu. Robust exponential stabilization for uncertain systems with state and control delay. International Journal of Systems Science, 34(12-13):675-682, October 2003.

S. Mondié and V.L. Kharitonov. Exponential estimates for retarded time-delay systems: an LMI approach. IEEE Trans. on Automatic Control, 50(2):268-273, 2005.

L. Moreau. Stability of continuous-time distributed consensus algorithms. In 43rd IEEE Conference on Decision and Control, 2004.

L. Moreau. Stability of multi-agent systems with timedependent communication links. IEEE Trans. on Automatic Control, 50(2):169-182, 2005.

U. Munz, A. Papachristodoulou, and F. Allgower. DelayDependent Rendezvous and Flocking of Large Scale MultiAgent Systems with Communication Delays. In 47th IEEE Conference on Decision and Control, 2008. CDC 2008, pages 2038-2043, 2008.

R. Olfati-Saber and R.M. Murray. Consensus problems in network of agents with switching topology and time delays. IEEE Trans. on Automatic Control, 49(9), 2004.

R. Olfati-Saber, A. Fax, and R.M. Murray. Consensus and cooperation in networked multi-agent systems. Proceedings of the IEEE, 95(1):215-233, 2007.

A. Seuret, M. Dambrine, and J.-P. Richard. Robust exponential stabilization for systems with time-varying delays. In 5th Workshop on Time Delay Systems, September 2004.

A. Seuret, D.V. Dimarogonas, and K.H. Johansson. Consensus under communication delays. In $47^{\text {th }}$ IEEE Conference on Decision and Control, 2008.

L. Xiao and S. Boyd. Fast linear iterations for distributed averaging. In 42th IEEE Conference on Decision and Control, 2003.

S. Xu, J. Lam, and M. Zhong. New exponential estimates for time-delay systems. IEEE Transactions on Automatic Control, 51(9):1501-1505, 2006. 